Tendências em Matemática Aplicada e Computacional, (2001), 13-22.

Sociedade Brasileira de Matemática Aplicada e Computacional.

\title{
Pavimentações Hiperbólicas
}

N.D. ALLAN, Departamento de Matemática, Instituto de Geociências e Ciências Exatas, UNESP, 13500-000 Rio Claro, SP, Brasil.

\section{Introdução}

O que entendemos por pavimentação de um plano geométrico por um polígono é uma cobertura do plano por cópias destes polígonos de modo que não haja buracos e que dois a dois destes polígonos tenham no máximo um lado em comum. Estamos essencialmente interessados em cobertura por triângulos. Obtemos a nossa pavimentação partindo de um triângulo fixo e refletindo-o indefinidamente sobre seus lados e sobre os lados de suas imagens. Estas reflexões formam um grupo que é o grupo de simetrias de nossa pavimentação. No plano euclidiano somente três tipos de triângulos pavimentam o plano: um triângulo equilátero, metade de um equilátero, e um triângulo retângulo isósceles. Como o produto de duas reflexões é uma rotação, os vértices do triângulo original serão centros de rotação de período inteiro cujos ângulos serão indicados respectivamente por $\{\pi / p, \pi / q, \pi / r\}$, e neste caso o grupo será indicado por $\{p, q, r\}$. Na geometria euclidiana $S=1 / p+1 / q+1 / r=1$ pois a soma dos ângulos internos de um triângulo é 180, ou $\pi$. Teremos os grupos $\{3,3,3\},\{2,3,6\}$ e $\{2,4,4\}$. Na Geometria Elítica cujo modelo é a superfície da esfera temos que $\mathrm{S}>1$ e só há um número finito de possibilidades para $\{\mathrm{p}, \mathrm{q}, \mathrm{r}\}$ : o grupo do tetraedro $\{2,3,3\}$, grupo do cubo $\{2,3,4\}$, e o do icosaedro, $\{2,3,5\}$. As outras pavimentações se realizam no plano hiperbólico pois aí $\mathrm{S}<1$. Existem vários modelos deste plano; escolhemos trabalhar no modelo de Poincaré. Vamos apresentar exemplos de pavimentações $\{2,3,7\},\{3,3,4\},\{3,4,4\}$ e $\{3,4,5\}$.

Afim de obtermos uma pavimentação com um número suficiente de triângulos vamos escrever as equações das reflexões e fazer suas composições. Necessitamos escrever um número razoável destas composições o que torna o problema complicado. Esbarramos no chamado problema das palavras num grupo: conhecemos seus geradores e relações e queremos calcular as palavras redundantes. Este é um problema muito difícil, porém no nosso caso ele simplifica-se pois o grupo é do tipo geométrico; seus elementos distintos são determinados pela ação em três pontos. Trataremos deste ponto mais adiante.

As pavimentações euclidianas nos acompanham desde a pré-história devido a necessidade que temos em decorar nosso ambiente. Elas apresentam uma variedade bem grande para trabalho com alunos bem como um campo com novos conceitos 
em geometria. Elas tem sido bastante estudadas e existem vários projetos, teses de Mestrado e de Doutorado em Educação matemática onde mostra-se seu aproveitamento para o ensino até do primeiro grau. Tanto as pavimentações elípticas como as hiperbólicas não são tão bem estudadas devido a dois aspectos: o primeiro é que elas envolvem transformações geométricas um pouco mais sofisticas, ou menos conhecidas que as reflexões e a segunda pela dificuldade de se construir fisicamente caleidoscópios nestas geometrias. As pavimentações hiperbólicas apareceram no inicio do século, com o estudo das superfícies de Riemann; H. Poincaré associou pavimentações às superfícies de Riemann. Os desenhos $\{2,3,7\}$ e $\{2,3,8\}$ aparecem em livros de F. Klein, sobre funções automorfas. M. Escher apresenta estas pavimentações em seus entalhes, como uma maneira do artista tratar o infinito. Na sala de aula, nos cursos de Graduação a Geometria Hiperbólica está cada vez mais ganhando seu espaço. O entendimento destas pavimentações começam a fazer parte da cultura matemática de um licenciado em Matemática. Mais recentemente há estudos sobre as aplicações destas pavimentações à teoria de códigos onde exemplos são bem vindos.

\section{Modelo de Poincaré para Geometria Hiperbólica}

Existem vários modelos de planos para a Geometria Hiperbólica: O modelo de Poincaré, o de plano de Klein - Cayley e o semiplano de Siegel.

$\mathrm{O}$ modelo de Poincaré é o seguinte: $\mathrm{O}$ plano é o interior $\mathrm{H}$ da circunferência $\mathrm{G}$ de centro na origem e raio um. As retas ou são segmentos diâmetros de $\mathrm{G}$ ou $\operatorname{arcos}$ de circunferências que são ortogonais a G. Distância é definida em termos da razão dupla; não faremos uso disto. O importante deste modelo é que a medida de ângulo entre duas retas hiperbólica que coincide com a medida de ângulo euclidiana. As congruências são produtos de reflexões hiperbólicas, e estas ou são reflexões lineares quando nossa reta hiperbólica é um diâmetro, ou são as chamadas inversões na circunferência que determina a reta hiperbólica. Em resumo, com estas noções de reta, distância, medida de ângulo e reflexão, podemos mostrar que elas tem as propriedades usuais da Geometria Euclidiana, com exceção da unicidade da paralela: por um ponto fora de uma reta podemos passar uma infinidade de retas paralelas a reta dada. O modelo de Siegel é equivalente a este modelo porem o plano é o semiplano cartesiano superior com retas sendo semicircunferências perpendiculares ao eixo real. $\mathrm{O}$ que vamos usar do modelo de Klein é que o plano é o interior $\mathrm{H}$ da circunferência $\mathrm{G}$ de centro na origem e raio um, ou mais geralmente o interior de uma cônica, e as retas são cordas de G.

\section{Ferramentas Hiperbólicas: Inversões}

Vamos agora escrever os primeiros comandos no "software" Mathematica com o objetivo de traçar a reta hiperbólica.

Comecemos com a fórmula da distância euclidiana: 
Vamos tratar de determinar o equação da circunferência que passa por três pontos dados, não colineares. Seu centro é eqüidistante aos três pontos, logo, como já foi explicado, temos o comando definido por

e seu raio é

Com isto podemos definir a transformação do plano chamada inversão numa circunferência $G^{*}$ de centro $C$ e raio r. A imagem de um ponto por esta transformação é dada pela seguinte construção. Para calcularmos a imagem de um ponto $P$, $\mathrm{P}$ fora de $\mathrm{G}^{*}$, traçamos por $\mathrm{P}$ uma tangente a $\mathrm{G}^{*}$. A imagem de $\mathrm{P}$ será a projeção ortogonal do ponto de tangência sobre a reta CP. A imagem será dada pela fórmula.

No caso particular de $\mathrm{G}^{*}=\mathrm{G}$ teremos.

$$
\wedge 2
$$

Esta transformação é o seu próprio inverso. Ela deixa fixo todo ponto de $\mathrm{G}^{*}$. Também deixa invariante retas que passam pela origem, leva circunferências que passam pela origem em retas, e leva as demais circunferências em outras circunferências.

Dados dois pontos $\mathrm{P}$ e $\mathrm{Q}$ no interior de $\mathrm{H}$, que não são colineares com $\mathrm{O}$, a reta hiperbólica $\ell$ é a circunferência que passa por $\mathrm{P}$ e $\mathrm{Q}$, e corta $\mathrm{G}$ ortogonalmente. A inversão em $\ell$ leva $\mathrm{G}$ nela mesma, e o mesmo acontece com $\mathrm{H}$. Um resultado simples de se demonstrar é que $\ell$ passa também pelo ponto . Daí seu circuncentro e circunraio serão dados pelas fórmulas :

Assim, a reta hiperbólica que passa por $\{\mathrm{P}, \mathrm{Q}\}$ é determinada por

Os pontos do infinito de dados por: são os pontos onde ela intercepta G, que são 


\section{Variação Angular e Reta Hiperbólica}

A seguir vamos determinar os limites de variação para traçarmos somente o trecho da circunferência entre $\mathrm{P}$ e Q.

Nosso comando será chamado de . Comecemos com

$$
\text { Sqrt }
$$

A direção do unitario da bissetriz do ângulo AQB será dada por

$\mathrm{O}$ valor do ângulo $\mathrm{AQB}$ é dado por

A seguir calculamos de uma maneira canônica o ângulo da bissetriz. A melhor maneira é usar o argumento complexo.

Finalmente

Agora estamos prontos para desenvolver a ferramenta mais importante de nosso trabalho: $\mathrm{O}$ comando que traça a reta hiperbólica e que chamaremos de

\section{Construção de Triângulos com Ângulos Dados}

Para efeito de cálculo, o que realmente necessitamos da Geometria Hiperbólica são as equações das reflexões hiperbólica bem como determinar as coordenadas dos vértices de um triângulo que tenha ângulos internos $\{\pi / p, \pi / q, \pi / r\}$, e isto será feito através de fórmulas da trigonometria hiperbólica. Uma das características desta geometria é que dois triângulos são congruentes se e somente se tem ângulos internos correspondentes iguais. Assim vamos colocar nosso triângulo com o vértice $\mathrm{C}$ na origem, A no eixo - $x$ e B numa reta que passa pela origem e faz um ângulo $\pi / r$ com o eixo $-x$. Os comandos que dão os lados do triângulo em função de seus ângulos internos são: 
Marquemos na reta que passa pela origem e tem inclinação $\mathrm{m} o$ pontos de distância dada d.

e daí os outros vértices tem coordenadas:

Estas funções se simplificam um pouco se o ângulo A for reto.

\section{Reflexões Hiperbólicas}

As respectivas reflexões são duas lineares e uma inversão:

Aqui reflH0 é a reflexão hiperbólica na reta hiperbólica que passa por P e Q, caso estes dois pontos não sejam colineares com $\mathrm{O}$.

Tendo o triângulo $\Delta$ e as equações das reflexões o nosso problema é um problema de cálculo.

\section{Palavras do Grupo $\{P, Q, R\}$}

Se não fosse pelas relações do grupo os cálculos seriam muito simples. Vamos exemplificar o que queremos dizer. Seja $a, b$, e c as reflexões que geram o grupo e seja e a identidade deste grupo. Assim $(a)^{2}=(b)^{2}=(c)^{2}=e$ bem como 
$(a b)^{r}=(b c)^{q}=(a c)^{p}=e . \quad\{\mathrm{a}, \mathrm{b}, \mathrm{c}\}$ chamam-se geradores e estas igualdades chamam-se relações deste grupo. Neste caso todo elemento do grupo é o produto de um certo número das letras $\{a, b, c\}$, e por analogia um tal produto chama-se uma palavra do grupo e o número de letras que a palavra tem chama-se comprimento da palavra. Em princípio é fácil fazer uma lista de todas as palavras de um determinado comprimento. Por exemplo se fizermos um lista de todas as palavras de comprimento dez sem aplicar as simplificações teremos 59049 tais palavras. Usando somente o fato de que são reflexões temos $(a)^{2}=(b)^{2}=(c)^{2}=e$, este número cai para um pouco mais de 17000 . Mesmo assim se conseguirmos fazer tal lista e aplicarmos estas 17000 palavras ao nosso triângulo ficamos decepcionados com o número de triângulos que iremos obter, cerca de 70. Nosso problema, depois da listagem, é um problema de otimização. Vamos primeiramente estudar o problema da listagem. Nossa experiência mostra que é mais fácil trabalhar com a lista ordenada dos elementos da palavra. O principio é simples: uma vez que temos todas as palavras de comprimento $\mathrm{n}$ basta adicionarmos ao final sucessivamente $\{\mathrm{a}, \mathrm{b}, \mathrm{c}\}$ e obteremos todas as palavras de comprimento $\mathrm{n}+1$. O problema das palavras é saber quando duas palavras representa o mesmo elemento do grupo. As relações $(a)^{2}=(b)^{2}=(c)^{2}=e$ nos permite em cada estágio desprezar as palavras cuja as duas últimas letras são iguais!

Nosso primeiro comando depende das letras iniciais, , de quantas letras $\mathrm{p}$ destas lista eu vou usar, $\mathrm{p}=3$ no nosso caso, e n é o comprimento das palavras geradas. No nosso caso list é $\{a, b, c\} \operatorname{logo} p=3$.

Observe que a repetição do comando serve para que o já calculado fique na memória do computador. O comando procede da seguinte maneira: Ao final do j1-ésimo elemento da lista já construída a nível n-1 anexamos via um novo $\{\mathrm{j} 2\}$ de . Obtemos uma lista dupla e a transformamos numa lista simples por meio do

Nossa próxima etapa é filtrar as relações de reflexão . O comando nos dá uma palavras genérica terminada em $\{\mathrm{n} 1, \mathrm{n} 1\}$. Assim por exemplo colocar após uma lista quer dizer que vou procurar elementos da lista da forma $\left\{{ }_{-},,_{-},,_{-}, \mathrm{n} 1, \mathrm{n} 1\right\} . \quad$ diz que primeiro tenho que formar o elemento da busca $\left\{{ }_{-},{ }_{-},{ }_{-},{ }_{-}, \mathrm{n} 1, \mathrm{n} 1\right\}$. Assim em cada estagio elimina as listas com os dois últimos elementos iguais

Para obtermos o novo comando procedemos como no eliminando as palavras que tem as duas letras finais repetidas, i.e., usando 
Obtemos:

O leitor pode se perguntar porque já tendo o comando

necessitamos de outro comando

e o motivo é economia de tempo bem como viabili-

dade. O gera $3^{10}=59049$ palavras de comprimento 10 , e o

reduz a umas 17000 palavras, o que ainda é muito.

\section{Cálculo das Imagens de $\Delta$}

O problema agora é calcular as imagens do nosso triângulo básico $\Delta$ e isto será feito pelo comando

- Todo o nosso argumento é baseado no fato de que uma isometria do plano é determinada por sua ação em três pontos não colineares. Vamos usar os três vértices de $\Delta$. Duas palavras de mesma ação nos vértices de $\Delta$ representa o mesmo elemento do grupo. Uma vez tendo a lista de palavras podemos calcular o elemento do grupo correspondente. A cada palavra associamos a composição das reflexões indicada nela . Primeiro substituímos $\{a, b, c\}$ pela lista de reflexões através de substitui, usando regra de substituição $->$.

Uma vez feita a substituição temos uma família de reflexões que deveremos compor, e isto é feito por meio do comando e a composição de todas as isometrias da lista é o último elemento deste resultado. Assim chegamos ao comando que depende da lista de isometrias isom e da lista de palavras, pala. no final é para que todas as componentes sejam calculadas.

No nosso caso específico o comando final que calcula a imagem dos vértices do nosso triângulo $\Delta$ será: 
O j é para sabermos qual é a aplicação que leva nosso triângulo original $\Delta$ num triângulo dado $\Delta 1$. é para bem ordenar nossa lista. Com o auxilio de podemos traçar um esboço da pavimentação para qualquer conjunto de palavras lty. Um gráfico pode ser obtido pelo comando:

onde é o traça o triângulo hiperbólico.

Nosso objetivo é selecionar uma palavra de um elemento do grupo que leva $\Delta$ em $\Delta 1$. Fazendo isto estaremos selecionando todas as palavras que representam elementos distintos do grupo.

Os resultados numéricos destes comandos são números flutuante o que não serve para fazer comparações. Afim de transforma-los em números racionais usamos o comando para aproximar todos os números a três casas decimais.

Começamos com o comando que reconhece elementos iguais de uma lista bem ordenada.

A seguir vamos jogar fora os elementos iguais. Primeiramente testamos se um elemento é igual ao próximo, e depois desprezamos os que são iguais ao posterior ficando somente o último elemento de uma cadeia de iguais. Adicionamos o último elemento da lista.

Usamos seguido de para comparar lista numéricas pois ele quase sempre não compara pontos flutuantes. Como já observamos este comando tem muitos triângulos repetidos e que a lista lty deve ser enxugada. Isto é feito refinando a escolha das palavras etapa por etapa, como foi feito em , só que agora tudo é mais delicado e complexo. Procedemos da seguinte maneira, usando o comando básico por trás de

Aplicamos nesta lista expandida. Note que ou é $\{0,1,2\}$ ou $\{a, b, c\}$. Excluímos as que dão elementos iguais, por meio de 
Este comando nos fornece no nível 10 algumas centenas de palavras o que já torna viável o traçado das figuras abaixo num computador pequeno.

nos da a lista dos triângulos distintos $\Delta 1$, juntamente com a última palavra que leva $\Delta$ em $\Delta 1$. Temos dois comandos. O primeiro nos fornece as coordenadas dos vértices dos triângulos obtidos neste estágio:

O segundo nos fornece a nova lista de palavras de comprimento $\mathrm{n}$.

\section{Mudança de Modelos}

Fechando nossa exposição vamos apresentar as funções que transformam o plano de Poincaré no plano de Klein e sua inversa. Geometricamente a aplicação

é obtida compondo-se a inversa da projeção estereográfica da esfera $\mathrm{S}$ sobre um plano $\mathrm{P}$, com a projeção ortogonal de S sobre P. Em coordenadas cartesianas ela é descrita como

$$
\begin{aligned}
& \wedge 2 \wedge 2 \wedge 0.5 \quad \wedge 2 \wedge 2 \wedge 0.5 \\
& \wedge 2 \\
& \wedge 2 \wedge 2 \quad \wedge 2
\end{aligned}
$$

Antes de encerrarmos devemos observar que há uma rotina do Cabri II que descreve pavimentações com triângulos refletindo um a um. Estes programas apresentam muitas coisas bonitas em Matemática sem dar uma idéia da matemática que está por trás. Estas pavimentações carregam com elas muita estrutura matemática, que deve ser informada aos alunos de graduação, apresentar como complemento a qualquer curso de variáveis complexas. Esperamos ser também útil aos estudantes de engenharia que trabalham na área de comunicações. Finalmente apresentamos alguns exemplos de pavimentações. 

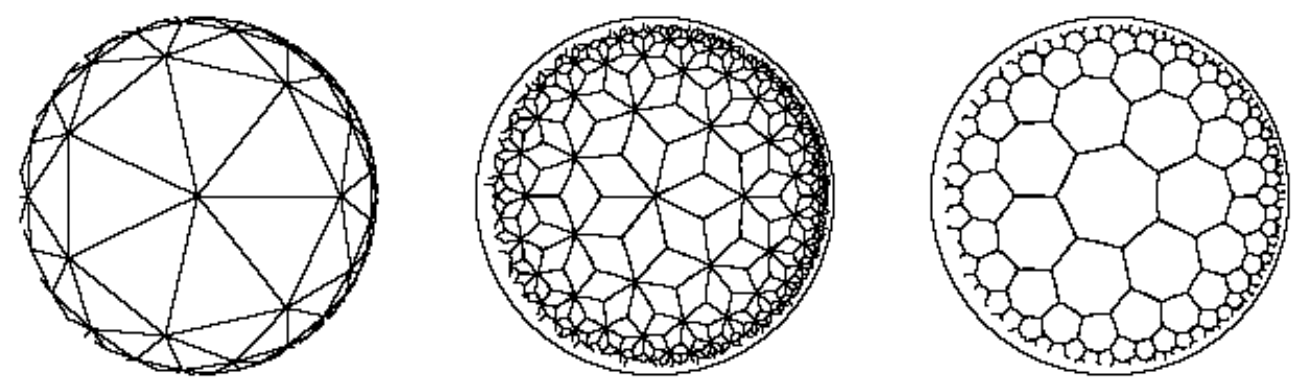

Figura 1: Subpavimentações de $\{2,3,7\}$
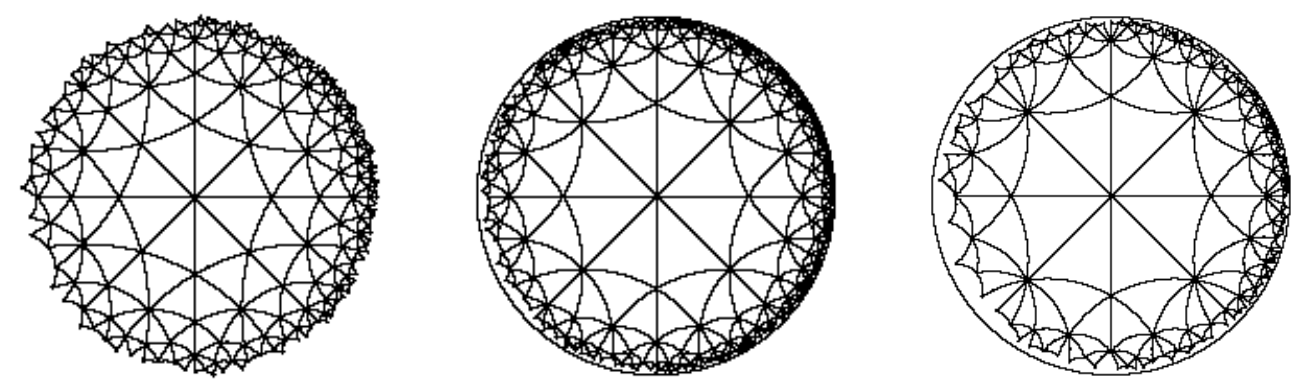

Figura 2: Pavimentações $\{3,3,4\},\{3,4,4\}$ e $\{3,4,5\}$

\section{Referências}

[1] N. Allan, Introdução ao Mathematica, Internet site: www.rc.unesp.br/ igce/matematica/nelo.

[2] M. Firer, Grupos Fuchsianos, notas de aula, IMECC Unicamp, 1999.

[3] G. Martin, "Foundations of the Euclidean and non Euclidean Geometry", Springer Verlag, New York. 